\title{
Evidence-based psychotherapy in children and adolescents: advances, methodological and conceptual limitations, and perspectives
}

\author{
Stefanie J. Schmidt • Benno G. Schimmelmann
}

Published online: 4 April 2013

(C) Springer-Verlag Berlin Heidelberg 2013

\section{Introduction}

Mental disorders have a high prevalence of up to $20 \%$ in children and adolescents worldwide and are often associated with substantial psychosocial impairments [1]. Many mental disorders with an early onset in childhood or adolescence have a high likelihood of persistence or recurrence of symptoms and are risk factors for other psychiatric disorders in adulthood [2]. Against this background, significant attention has been paid to the development of psychological therapies for children and adolescents that aim to reduce current symptom levels and their detrimental effects on functioning and developmental trajectories.

The earliest reviews of these psychological therapies included studies with mostly low methodological quality. They found that the rate of improvement did not differ significantly whether or not any type of psychotherapy was provided. The authors $[3,4]$ emphasized the need for more stringent standards in intervention research and more specified, well-documented treatments. This led to an increase in randomized controlled trials (RCTs) which demonstrated that psychotherapy can indeed produce significant benefit compared to control conditions (e.g. [5-7]). These results have coalesced into evidence-based treatments for children and adolescents applicable to several diagnostic categories including anxiety disorders, depressive disorders, eating disorders, autism spectrum disorders, substance abuse, and attention deficit hyperactivity disorders $[8,9]$. The majority of these treatments under study in

S. J. Schmidt ( $\square) \cdot$ B. G. Schimmelmann

University Hospital of Child and Adolescent Psychiatry,

University of Bern, Bolligenstrasse 111,

3000 Bern 60, Switzerland

e-mail: stefanie.schmidt@kjp.unibe.ch this age group can be described by the umbrella term 'cognitive-behavioural therapy' (CBT); they include both programmes designed specifically for children and adolescents and generic approaches for all age groups such as CBT for anxiety disorders $[8,10]$. With the number of evidence-based treatments expanding, research has started to draw on the relative efficacy of treatments for specific subtypes of disorders [10] and different intervention techniques for the same disorder [11]. For example, the review by Leenarts et al. [11] in this issue reported that traumafocused CBT techniques revealed better empirical support for the treatment of children exposed to maltreatment than both non-trauma focused methods and trauma-specific treatments for children and adolescents with comorbid disorders. Recent studies also investigated combined effects of psychopharmacologic and psychotherapeutic interventions but found no consistent evidence favouring integrated interventions (e.g. [12]). In summary, child and adolescent psychotherapy research has shown remarkable progress towards evidence-informed treatments in recent years. However, it seems important to move on to surmount methodological and conceptual limitations.

\section{Methodological aspects}

Despite the increase in the quantity of evaluation studies, child and adolescent psychiatry is still far from having a knowledgebase sufficient for creating evidence-informed decisions about psychotherapy for all mental disorders. This is at least partially due to the low methodological quality of the majority of these studies. Therefore, the conclusions of recent meta-analyses and reviews of treatment efficacy for given disorders were often preliminary or inconclusive, because the number of included RCTs was 
generally small, often with low statistical power and a high or even unknown risk for biased sample selection and randomization procedures $[10,13,14]$. In addition, most of these studies suffered from selective outcome reporting, and about one-third of the published studies included a follow-up assessment with a mean time-lag of 5 months after therapy only [8, 14]. Therefore, the durability of treatment effects remains largely unclear. As a consequence, we do not know yet whether treatment gains could be maintained by additional treatments or booster-sessions.

This underlines the need for larger, methodologically sound RCTs assessing long-term effects. In addition, costbenefit analyses may highlight psychotherapy's potential to reduce the direct and indirect costs associated with child and youth mental health problems by alleviating psychosocial impairments.

Current assessments of therapeutic change are often limited to symptom domains and are mostly administered in a highly standardized setting in the laboratory. Treatment effects, however, should also generalize to different facets of the problem (e.g. cognitive, emotional, social, behavioural aspects) and to different contexts in everydaylife [15]. Therefore, one measure or informant is not able to capture all of these aspects of treatment efficacy. Another limitation of current outcome assessment in child and adolescent psychotherapy research is the reliance on few and often non-blinded informants with low inter-informant agreement across settings, for example, teachers and parents [14, 16, 17]. This may lead to biased or inconsistent information about treatment effects. A key issue in the assessment of treatment effects is whether improvements found in standardized, nomothetic measures that place the individual's functioning in a normative context are also clinically relevant for the individual patient. Idiographic assessments that take the individual patterns of change and the individual developmental state into account are largely ignored in the current literature [18].

Therefore, multiple measures and multiple informants are needed to evaluate different aspects of the problem. Moreover, symptom and diagnostic measures should be supplemented by measures of real world-functioning across various contexts like direct observation of the patient's behaviour (e.g. behaviour in school, at home, with friends). The event sampling method (ESM) [19] may be a promising measure of real world-functioning, because it allows researchers to study individual experiences that vary across time in their natural environment. As ESM minimizes recall biases by the event-contingent assessment, it may also be adequate for children with low levels of cognitive functioning, who are otherwise often excluded from studies. Newly developed person-focused assessments [20] address the shortcomings of standardized, nomothetic measures. They seem to be an efficient strategy for identifying intra- individual changes in personally relevant treatment goals by repeatedly assessing the problems that patients and their care-givers consider to be most important.

The application of several measures and informants, however, raises the question of how to deal with inconsistent findings. For example, what if some measures support treatment efficacy but others do not? This question has not been adequately addressed, because many researchers tried to circumvent discussing inconsistent findings by focussing only on significant outcomes or by using terms such as 'probably effective' or 'almost evidence-based' [21]. It seems important to develop clear standards on how to integrate conflicting results based on multiple outcome domains and outcome measures and how to interpret them in terms of treatment efficacy.

The range of possible changes (RPC) model [22] may be a helpful framework to this end, as it has been designed to identify and integrate within- and between-study consistencies in a systematic way. Future studies are needed to assess the reliability and validity of RPC's proposed classification scheme and the relevance of its clinical implications.

Meta-analyses and reviews indicate that many kinds of psychotherapy produce therapeutic effects in children and adolescents $(9,34)$. However, more in depth-analyses of treatment effects are necessary to identify under what conditions these treatments work best (i.e. moderators; e.g. gender, therapy setting and age) and to investigate how they lead to change (i.e. mediators; e.g. therapeutic alliance and self-efficacy expectations) [24, 25]. For example, few treatments have been adapted to the special needs of preschool children [10]. Assessing the moderating effect of age may have important implications towards a differential indication of these interventions and may emphasize the need to tailor therapies to the developmental stage of participants. Mediation analysis may shed light on change mechanisms on a cognitive [26], behavioural [25], and a neuronal level [27] as well as their interactions. Mediation analysis may also be an appropriate statistical method to test critical components of multimodal treatments. Identifying possible mechanisms of change would have important clinical implications. Therapists could optimize effects of current treatments by focusing on change mechanisms that need to be targeted [28].

\section{Conceptual aspects}

Only a few manuals have been specifically developed for children or adolescents. Many CBT treatments for children and adolescents can be viewed as downward extensions of intervention programmes originally designed for adult patients [29]. This was done based on the assumption that 
the underlying theoretical models are directly generalizable to younger age groups, but this has rarely been formally tested. This assumption implies that patients with the same diagnosis would all benefit from the same intervention across the lifespan. However, this is not congruent with empirical evidence reporting, e.g. smaller effect sizes for children than for adolescents [30].

This highlights the need for studies to test the validity of the underlying models and mechanisms of change across different age groups. For example, it is important to gain a precise understanding of the cognitive, emotional and interpersonal prerequisites of the numerous intervention techniques used in child and adolescent psychotherapy. This may help in choosing the techniques most appropriate to the current developmental state of an individual rather than age per se.

A range of evidence-based treatments have been developed in the past, however, they were only rarely incorporated into clinical practice [31], and their efficacy was substantially reduced when they were tested under clinically representative general care conditions [23]. Possible implementation barriers accounting for these differences may be that patients in service settings have higher rates of comorbidities and more psychosocial impairments as well as motivational problems. It is also possible that different mechanisms leading to a respective mental disorder are present in routine clinical care than in research settings, thus rendering deviations from treatment protocols mandatory [23]. These differences raise concerns that treatments tested in RCTs may not be applicable to patients treated in routine clinical care [32], because the adherence to a predefined sequence of sessions with predetermined therapy contents in RCTs may constrain the therapist's ability to adapt each therapy session to the individual treatment needs of a patient.

To address these concerns, modular [33] or stepped-care models may be promising approaches. As more individualized approaches they may facilitate the coverage of comorbid problems and sequential changes in treatment response without a substantial loss of internal validity. Evaluation of such treatment concepts in child and adolescent psychiatry is recommended to bridge the gap between efficacy and effectiveness.

\section{Conclusions}

In summary, psychotherapy research in children and adolescents has generated several evidence-based interventions in recent years. At this point, we know much about what outcomes are produced by treatments but have little understanding what drives these changes. The need for individualization in assessment and therapy is not new but still in its infancy. Furthermore, treatments should be adapted to a patient's developmental level to optimize treatment efficacy. Novel assessment methods and trial designs that take individual treatment needs and the context of problems into account seem promising to personalize evidence-based treatments and thereby facilitate their implementation into clinical practice.

\section{References}

1. Kieling C, Baker-Henningham H, Belfer M et al (2011) Child and adolescent mental health worldwide: evidence for action. Lancet 378:1515-1525

2. Flisher AJ, Hatherill S, Dhansay Y (2010) Specific mental health disorders: child and adolescent mental disorders. In: Patel V, Woodward A, Feigin VL, Heggenhougen HK, Quah S (eds) Mental and neurological public health. A global perspective. Elsevier, San Diego, pp 303-310

3. Levitt EE (1957) The results of psychotherapy with children: an evaluation. J Consult Psychol 21:189-196

4. Levitt EE (1963) Psychotherapy with children: a further evaluation. Behav Res Ther 60:326-329

5. Layne CM, Salzman WR, Poppleton L et al (2008) Effectiveness of a school-based group psychotherapy program for war-exposed adolescents: a randomized controlled trial. J Am Acad Child Adolesc Psychiatry 47(9):1048-1062

6. Baghdadli A, Brisot J, Henry V et al (2013) Social skills improvement in children with high functioning autism: a pilot randomized controlled trial. Eur Child Adolesc Psychiatry. doi: 10.1007/s00787-013-0388-8

7. Ruble LA, McGrew JH, Toland MD, Dalrymple NJ, Jung LA (2013) A randomized controlled trial of COMPASS web-based and face-to-face teacher coaching in autism. J Consult Clin Psychol. doi:10.1037/a0032003

8. Weisz JR, Kazdin AE (2010) Evidence-based psychotherapies for children and adolescents. Guilford, New York

9. Chorpita BF, Daleiden EL, Ebesutani C et al (2011) Evidencebased treatments for children and adolescents: an updated review of indicators of efficacy and effectiveness. Clin Psychol Sci Pract 18(2):154-172

10. Reynolds S, Wilson C, Austin J, Hooper L (2012) Effects of psychotherapy for anxiety in children and adolescents: a metaanalytic review. Clin Psychol Rev 32:251-262

11. Leenarts LEW, Diehle J, Doreleijers TAH, Jansma EP, Lindauer RJL (2013) Evidence-based treatments for children with traumarelated psychopathology. Eur Child Adolesc Psychiatry. doi: 10.1007/s00787-012-0367-5

12. Hetrick SE, Cox GR, Merry SN (2011) Treatment-resistant depression in adolescents: is the addition of cognitive behavioral therapy of benefit? Psychol Res Behav Manag 4:97-112

13. Storeb $\varnothing$ OJ, Skoog M, Damm D et al (2011) Social skills training for attention deficit hyperactivity disorder (ADHD) in children aged 5 to 18 years. Cochrane DB Syst Rev 12. doi:10.1002/ 14651858.CD008223.pub2

14. Zwi M, Jones H, Thorgaard $\mathrm{C}$ et al (2011) Parent training interventions for attention deficit hyperactivity disorder (ADHD) in children aged 5 to 18 years (Review). Cochrane DB Syst Rev: 12. doi:10.1002/14651858.CD003018.pub3

15. Kazdin AE (2005) Evidence-based assessment for children and adolescents: issues in measuring development and clinical application. J Clin Child Adolesc Psychol 34(3):548-558 
16. Ebesutani C, Bernstein A, Martinez JI, Chorpita BF, Weisz JR (2011) The youth self-report: applicability and validity across younger and older youths. J Clin Child Adolesc Psychol 40(2):338-346

17. Rescorla LA, Ginzburg S, Achenbach TM et al (2013) Crossinformant agreement between parent-reported and adolescent self-reported problems in 25 societies. J Clin Child Adolesc Psychol 42(2):262-273

18. Mohr C, Schneider S (2013) Anxiety disorders. Eur Child Adolesc Psychiatry 22(1):17-22

19. Reis HT, Gable SL (2000) Event-sampling and other methods for studying everyday experience. In: Reis HT, Judd CM (eds) Handbook of research methods in social and personality psychology. Cambridge University Press, New York, pp 190-222

20. Weisz JR, Chorpita BF, Frye A et al (2011) Youth top problems: using idiographic, consumer-guided assessment to identify treatment needs and to track change during psychotherapy. J Consult Clin Psychol 79(3):369-380

21. De Los Reyes A, Kazdin AE (2008) When the evidence says, "yes, no, and maybe so": attending to and interpreting inconsistent findings among evidence-based interventions. Curr Dir Psychol Sci 17(1):47-51

22. De Los Reyes A (2009) Identifying evidence-based interventions for children and adolescents using the range of possible changes model: a meta-analytic illustration. Behav Modif 33(5):583-617

23. Weisz JR, Ugueto AM, Cheron DM, Herren J (2013) Evidencebased youth psychotherapy in the mental health ecosystem. J Clin Child Adolesc Psychol 42(2):274-286

24. Nilsen TS, Eiseman M, Kvernmo S (2012) Predictors and moderators of outcome in child and adolescent anxiety and depression: a systematic review of psychological treatment studies. Eur Child Adolesc Psychiatry 22(2):69-87

25. Webb CA, Aurebach RP, DeRubeis RJ (2012) Process of change in CBT of adolescent depression: review and recommendations. J Clin Child Adolesc Psychol 41(5):654-665
26. Wollburg E, Meyer B, Osen B, Löwe B (2013) Psychological change mechanisms in anorexia nervosa treatments: how much we know? J Clin Psychol. doi:10.1002/jclp.21945

27. Eack SM, Pogue-Geile MF, Greenwald DP, Hogarty SS, Keshavan MS (2010) Mechanisms of functional improvement in a 2-year trial of enhancement therapy for early schizophrenia. Psychol Med 41(6):1-9

28. Schmidt SJ, Mueller DR, Roder V (2011) Social cognition as a mediator variable between neurocognition and social cognition in schizophrenia: empirical review and new results by structural equation modeling. Schiz Bull 37(2):41-54

29. Frankel SA, Gallerani CM, Garber J (2012) Developmental considerations across childhood. In: Szightey E, Weisz JR, Findling RL (eds) Cognitive-behavior therapy for children and adolescents. American Psychiatric Publishing, Arlington, pp 29-74

30. Weisz JR, Weiss B, Han S, Granger DA, Morton Z (1995) Effectiveness of psychotherapy with children and adolescents revisited: a meta-analysis of treatment outcome studies. Psychol Bull 117:450-468

31. Westen D, Novotny CM, Thompson-Brenner H (2004) The next generation of psychotherapy research: Reply to Ablon and Marci (2004), Goldfried and Eubanks-Carter (2004) and Haaga (2004). Psychol Bull 130(4):631-683

32. Kovshoff H, Williams S, Vrijens M et al (2012) The decisions regarding ADHD management (DRAMa) study: uncertainties and complexities in assessment, diagnosis and treatment, from the clinician's point of view. Eur Child Adolesc Psychiatry 21:87-99

33. Weisz JR, Chorpita BF, Palinkas LA et al (2012) Testing standard and modular designs for psychotherapy treating depression, anxiety, and conduct problems in youth. Arch Gen Psychiatry 69(3):274-282 